\title{
MAXWELL FIELDS IN SPACETIMES ADMITTING NON-NULL KILLING VECTORS
}

\author{
István Rácz \\ MTA KFKI Research Institute for Particle \& Nuclear Physics \\ H-1525 Budapest 114, P.O.B. 49, Hungary
}

\begin{abstract}
Abstrac: We consider source-free electromagnetic fields in spacetimes possessing a non-null Killing vector field, $\xi^{a}$. We assume further that the electromagnetic field tensor, $F_{a b}$, is invariant under the action of the isometry group induced by $\xi^{a}$. It is proved that whenever the two potentials associated with the electromagnetic field are functionally independent the entire content of Maxwell's equations is equivalent to the relation $\nabla^{a} T_{a b}=0$. Since this relation is implied by Einstein's equation we argue that it is enough to solve merely Einstein's equation for these electrovac spacetimes because the relevant equations of motion will be satisfied automatically. It is also shown that for the exceptional case of functionally related potentials $\nabla^{a} T_{a b}=0$ implies along with one of the relevant equations of motion that the complementary equation concerning the electromagnetic field is satisfied.

PACS numbers: 04.20.Cv, 04.20.Me, 04.40.+c
\end{abstract}

In the framework of general relativity the spacetime geometry, which serves as a stage for the history of physical fields, is influenced by the matter content of the spacetime. More definitely, the geometry of a spacetime is related to the matter distribution by Einstein's equation

$$
R_{a b}-\frac{1}{2} g_{a b} R=8 \pi T_{a b}
$$

where $R_{a b}$ denotes the Ricci tensor associated to $g_{a b}$ and $T_{a b}$ is the energy-momentum tensor of matter fields. In fact, when one is looking for spacetimes which are solutions of Einsten's equation with selected sort of matter fields beside eq. (1) one has to consider simultaneously the relevant Euler-Lagrange equations governing the evolution of matter fields, as well. In general, however, these equations of motion for the matter fields and Einstein's equation give rise in a selected local coordinate system to a complicated system of coupled non-linear second order partial differential equations for the components of the spacetime metric and the tensor fields 
representing the matter fields in the spacetime. On the other hand, Einstein's equation - or more precisely, its "integrability condition", the twice contracted Bianchi identity - implies the relation

$$
\nabla^{a} T_{a b}=0
$$

which contains a great deal of information on the behaviour of matter fields. More definitely, the above relation gives rise, in general, to algebraical relationships between various terms of the relevant equations of motion (see, for instance, Ref. [1]), and so Einstein's equation implies along with some of the Euler-Lagrange equations that the complementary equations of motion for the matter fields are satisfied. In particular, the Euler-Lagrange equations are for a perfect fluid source equivalent to eq. (2). Moreover, it can easily be checked that the whole content of the equations of motion is also equivalent to the above relation for an arbitrary real scalar field. Hence, for these sort of matter fields it is enough to postulate the form of the energy-momentum tensor, $T_{a b}$, and solve Einstein's equation alone because then the relevant equations of motion concerning the evolution of these matter fields are automatically satisfied.

The purpose of this paper is to show that in a spacetime, $\left(M, g_{a b}\right)$, possessing a non-null Killing vector field, $\xi^{a}$, and an antisymmetric tensorfield, $F_{a b}$, representing a source-free electromagnatic field invariant under the action of the isometry group induced by $\xi^{a}$ Maxwell's equations are just consequences of the relation $\nabla^{a} T_{a b}=0$ whenever the associated two potentials are functionally independent. Hence, in particular, for these electrovac spacetimes it is also enough to prescribe the form of $T_{a b}$ known for source-free electromagnetic fields and solve then the relevant Einstein's equation. The exceptional case of functionally related potentials is also considered and it is shown that the relation $\nabla^{a} T_{a b}=0$ together with half of the relevant equations of motion imply that the complementary equation holds.

Before giving the proof of the above statements we recall some notions and results. In a spacetime, $\left(M, g_{a b}\right)$, the electromagnetic field can be represented by an antisymmetric tensor field, $F_{a b}$, and the electric and magnetic fields measured by an observer with 4 -velocity, $u^{a}$, are given as $[2]$

$$
E_{a}=F_{a b} u^{b} \quad \text { and } \quad B_{a}=-\frac{1}{2} \epsilon_{a b c d} u^{b} F^{c d},
$$

where $\epsilon_{a b c d}$ denotes the volume element associated with $g_{a b}$. The Maxwell's equations for a source-free electromagnetic field take the following form

$$
\begin{aligned}
& \nabla^{a} F_{a b}=0, \\
& \nabla_{[a} F_{b c]}=0,
\end{aligned}
$$


where $\nabla^{a}$ denotes the covariant derivative operator determined by $g_{a b}$. Since we have a preferred non-null vector field, $\xi^{a}$, on $M$ it is advantageous to apply the following decomposition of $F_{a b}$ :

$$
F_{a b}=-\left(\xi^{e} \xi_{e}\right)^{-1}\left\{2 \xi_{[a} \Psi_{b]}+\epsilon_{a b c d} \xi^{c} \Omega^{d}\right\}
$$

where

$$
\Psi_{a}=F_{a b} \xi^{b} \quad \text { and } \quad \Omega_{a}=-\frac{1}{2} \epsilon_{a b c d} \xi^{b} F^{c d} .
$$

Note that whenever the Killing field, $\xi^{a}$, is timelike $\Psi_{a}$ (resp. $\Omega_{a}$ ) is proportional to the electric (resp. magnetic) field measured by observers moving along the Killing trajectories.

To present a more substantial description of the electromagnetic field it is worth introducing the notion of duality. The dual of a 2 -form, $X_{a b}$, is defined as

$$
\widetilde{X}_{a b}=\frac{1}{2} \epsilon_{a b c d} X^{c d}
$$

Then, $\Omega_{a}$ can be given in terms of $\xi^{a}$ and $\widetilde{F}_{a b}$ as

$$
\Omega_{a}=-\widetilde{F}_{a b} \xi^{a}
$$

as well as, eqs. (4) and (5) can be recast into the equivalent form

$$
\begin{aligned}
& \nabla_{[a} \widetilde{F}_{b c]}=0, \\
& \nabla^{a} \widetilde{F}_{a b}=0 .
\end{aligned}
$$

Consequently, the Maxwell's equations are for a source-free electromagnetic field equivalent to either of the pairs of eqs. (4) and (11), or (5) and (10).

Consider, now, the second pair of these equations, i.e.,

$$
\begin{aligned}
& \nabla_{[a} \widetilde{F}_{b c]}=0, \\
& \nabla_{[a} F_{b c]}=0 .
\end{aligned}
$$

Remember that we have a preferred vector field, $\xi^{a}$, on the spacetime and so, by the application of the projection operator, $h^{b}{ }_{a}$, associated with $\xi^{a}$, i.e., the operator $h^{b}{ }_{a}=\delta^{b}{ }_{a}-\left(\xi^{e} \xi_{e}\right)^{-1} \xi^{b} \xi_{a}$, we may uniquely decompose the 3 -form, $\nabla_{[a} F_{b c]}$, into 3 -forms such that they have definite "tangential" or "perpendicular" character with regard to their free indices. Clearly, $\nabla_{[a} F_{b c]}$ coincides then with the zero 3 -form if and only if its various types of projections vanish. It is easy to check that there are only two non-identically vanishing projections of $\nabla_{[a} F_{b c]}$, namely, 
$\nabla_{[e} F_{f g]} h_{a}^{e} h_{b}^{f} h_{c}^{g}$ and $\nabla_{[e} F_{f g]} h_{a}^{e} h_{b}^{f} \xi^{g}$ or, equivalently, $\nabla_{[a} F_{b e]} \xi^{e}$. Therefore Maxwell's equations are equivalent to the following set of equations

$$
\begin{gathered}
\nabla_{[e} F_{f g]} h_{a}^{e} h_{b}^{f} h^{g}{ }_{c}=0, \\
\nabla_{[e} \widetilde{F}_{f g]} h^{e}{ }_{a} h^{f}{ }_{b} h^{g}{ }_{c}=0, \\
\nabla_{[a} F_{b e]} \xi^{e}=0, \\
\nabla_{[a} \widetilde{F}_{b e]} \xi^{e}=0 .
\end{gathered}
$$

It is well-known that, for an arbitrary 2 -form, $X_{a b}$, the identity

$$
2 \nabla_{[a} \Phi_{b]}=3 \nabla_{[a} X_{b e]} \xi^{e}-\mathcal{L}_{\xi} X_{a b}
$$

with $\Phi_{a}=X_{a b} \xi^{b}$ is satisfied, where $\mathcal{L}_{\xi} X_{a b}$ denotes the Lie derivative of $X_{a b}$ with respect to $\xi^{a}$. Applying this identity for the 2-forms, $F_{a b}$, and , $\widetilde{F}_{a b},-$ which are, according to our assumption, invariant under the action of the isometry group associated with the Killing field, $\xi^{a}$, - we get that eqs. (16) and (17) are satisfied if and only if the 1-forms, $\Psi_{a}$, and, $\Omega_{a}$, defined by eq. (7) are closed, i.e., whenever there are functions at least locally, $\Psi$, and, $\Omega$, such that $\Psi_{a}=\nabla_{a} \Psi$ and $\Omega_{a}=\nabla_{a} \Omega$. Note that, as a consequence of the definition $\Psi_{a}$ and $\Omega_{a}$, we have

$$
\mathcal{L}_{\xi} \Psi=\mathcal{L}_{\xi} \Omega=0
$$

The remaining two equations of motion, i.e., eqs. (14) and (15), can be shown to be equivalent to

$$
\begin{aligned}
& \left(\nabla^{a} F_{a b}\right) \xi^{b}=0, \\
& \left(\nabla^{a} \widetilde{F}_{a b}\right) \xi^{b}=0,
\end{aligned}
$$

from which, by the substitution of the decomposition of $F_{a b}$ in terms of $\Psi, \Omega$ and $\xi^{a}$, we obtain $[3,4]$

$$
\begin{aligned}
& \nabla^{a} \nabla_{a} \Psi-v^{-1}\left\{\left(\nabla^{a} \Psi\right)\left(\nabla_{a} v\right)+\left(\nabla^{a} \Omega\right) \omega_{a}\right\}=0, \\
& \nabla^{a} \nabla_{a} \Omega-v^{-1}\left\{\left(\nabla^{a} \Omega\right)\left(\nabla_{a} v\right)-\left(\nabla^{a} \Psi\right) \omega_{a}\right\}=0,
\end{aligned}
$$

where $v=\xi^{e} \xi_{e}$ and $\omega^{a}$ denotes the twist vector of $\xi^{a}$, i.e., $\omega^{a}=\epsilon^{a b c d} \xi_{b} \nabla_{c} \xi_{d}$.

Summarizing the above results we can say that in a spacetime possessing a non-null Killing vector field, $\xi^{a}$, a 2 -form, $F_{a b}$, which is invariant under the action of the isometry group induced by the Killing field satisfies Maxwell's equations for a source-free electromagnetic field if and 
only if there exist at least locally two functions, $\Psi$, and, $\Omega$, on $M$ such that $\mathcal{L}_{\xi} \Psi=\mathcal{L}_{\xi} \Omega=0$ and

$$
F_{a b}=-2 v^{-1}\left\{\xi_{[a} \nabla_{b]} \Psi+\left(\xi_{[a} \nabla_{b]} \Omega\right)^{\sim}\right\},
$$

as well as, eqs. (22) and (23) are satisfied.

In the remaining part of this paper - using the above formulation of electrovac fields in spacetimes admitting a non-null Killing vector field - we are going to examine how strong restrictions on the relevant equations of motion can be derived from the relation $\nabla^{a} T_{a b}=0$.

Consider, now, the energy-momentum tensor of an electrovac Maxwell field, i.e.,

$$
T_{a b}=\frac{1}{4 \pi}\left\{F_{a c} F_{b}^{c}-\frac{1}{4} g_{a b} F_{e d} F^{e d}\right\} .
$$

The substitution of the decomposition of $F_{a b}$ in terms of $\Psi, \Omega$ and $\xi^{a}$ into eq. (25) yields

$$
\begin{aligned}
T_{a b}=\frac{v^{-2}}{4 \pi}\{ & {\left[\left(\nabla^{e} \Psi\right)\left(\nabla_{e} \Psi\right)+\left(\nabla^{e} \Omega\right)\left(\nabla_{e} \Omega\right)\right]\left(\xi_{a} \xi_{b}-\frac{1}{2} v g_{a b}\right) } \\
& \left.+v\left[\left(\nabla_{a} \Psi\right)\left(\nabla_{b} \Psi\right)+\left(\nabla_{a} \Omega\right)\left(\nabla_{b} \Omega\right)\right]+2 \xi_{(a} \epsilon_{b) c g h}\left(\nabla^{c} \Psi\right) \xi^{g}\left(\nabla^{h} \Omega\right)\right\} .
\end{aligned}
$$

Then, using the fact that $\xi^{a}$ is a Killing field - and so, for instance, $\nabla_{(a} \xi_{b)}=\xi^{a} \nabla_{a} v=\nabla^{a} \xi_{a}=0$ - , furthermore, that $\mathcal{L}_{\xi} \Psi=\mathcal{L}_{\xi} \Omega=0$, by a straightforward calculation we obtain

$$
\begin{aligned}
\nabla^{a} T_{a b}=\frac{v^{-1}}{4 \pi}\{ & {\left[\nabla^{a} \nabla_{a} \Psi-v^{-1}\left(\nabla^{a} \Psi\right)\left(\nabla_{a} v\right)\right]\left(\nabla_{b} \Psi\right) } \\
+ & {\left.\left[\nabla^{a} \nabla_{a} \Omega-v^{-1}\left(\nabla^{a} \Omega\right)\left(\nabla_{a} v\right)\right]\left(\nabla_{b} \Omega\right)\right\}+\frac{1}{4 \pi} \nabla^{a}\left[2 v^{-2} \xi_{(a} \Sigma_{b)}\right], }
\end{aligned}
$$

where $\Sigma^{a}=\epsilon^{a b c d}\left(\nabla_{b} \Psi\right) \xi_{c}\left(\nabla_{d} \Omega\right)$ which is for the case of a timelike Killing field proportional to the Poynting vector, $P^{a}=\frac{1}{2}(-v)^{-1 / 2} \epsilon^{a b c d} E_{b} B_{c} \xi_{d}$, of the electromagnetic field measured by observers moving along the Killing trajectories.

Note that whenever $\left(\nabla_{[a} \Psi\right)\left(\nabla_{b]} \Omega\right)=0$ holds in an open region, i.e., $\nabla_{a} \Psi$ and $\nabla_{a} \Omega$ are proportional, $\Sigma_{a}$ vanishes there identically. Then, either $\Psi$ or $\Omega$ is constant, or neither $\nabla_{a} \Psi$ nor $\nabla_{a} \Omega$ vanishes in the selected open region. In the first case, eq. (27) simplifies into

$$
\nabla^{a} T_{a b}=\frac{v^{-1}}{4 \pi}\left[\nabla^{a} \nabla_{a} \Phi-v^{-1}\left(\nabla^{a} \Phi\right)\left(\nabla_{a} v\right)\right]\left(\nabla_{b} \Phi\right),
$$

where $\Phi$ denotes either of the fields, $\Psi$, or, $\Omega$, according to that we are considering a pure "electric" or "magnetic" field. This conclusion completes the proof of our conjecture for this particular case. For the second case, note that if there is an open region where $\nabla_{a} \Psi$ and $\nabla_{a} \Omega$ are non-vanishing the potentials, $\Psi$ and $\Omega$, are functionally related. Then, it can easily be seen that $\left(\nabla_{b} \Omega\right) \omega_{a}\left(\nabla^{a} \Psi\right)-\left(\nabla_{b} \Psi\right) \omega_{a}\left(\nabla^{a} \Omega\right)=0$ holds which yields along with eq. (27)

$$
\begin{aligned}
\nabla^{a} T_{a b}=\frac{v^{-1}}{4 \pi}\{ & {\left[\nabla^{a} \nabla_{a} \Psi-v^{-1}\left\{\left(\nabla^{a} \Psi\right)\left(\nabla_{a} v\right)+\omega_{a} \nabla^{a} \Omega\right\}\right]\left(\nabla_{b} \Psi\right) } \\
+ & {\left.\left[\nabla^{a} \nabla_{a} \Omega-v^{-1}\left\{\left(\nabla^{a} \Omega\right)\left(\nabla_{a} v\right)-\omega_{a} \nabla^{a} \Psi\right\}\right]\left(\nabla_{b} \Omega\right)\right\} . }
\end{aligned}
$$


Hence for this particular case, the relation $\nabla^{a} T_{a b}=0$ alone does not imply that Maxwell's equations are satisfied. Nevertheless, we may economize the content of eq. (29) by arguing that the relation $\nabla^{a} T_{a b}=0$ together with one of eqs. (22) and (23) always implies that the complementary equation of motion is automatically satisfied.

For the remaining part of this proof, we shall assume that $\nabla_{a} \Psi$ and $\nabla_{a} \Omega$ are linearly independent. Note that in an open region of $M$ where $\nabla_{a} \Psi$ and $\nabla_{b} \Omega$ are linearly independent the four 4-vectors, $\xi^{a}, \nabla^{a} \Psi, \nabla^{a} \Omega$ and $\Sigma^{a}$, give rise to a well-defined basis field, $\left\{\xi^{a}, \nabla^{a} \Psi, \nabla^{a} \Omega, \Sigma^{a}\right\}$, provided that $\Sigma^{a}$ is not a null vector field which may occur when $\xi^{a}$ is spacelike. If $\Sigma^{a}$ happens to be null it can be shown that either $\nabla^{a} \Psi$ or $\nabla^{a} \Omega$ has to be parallel to $\Sigma^{a}$. Denote by $\Phi$ the one from $\Psi$ and $\Omega$ for which $\nabla^{a} \Phi$ is proportional to $\Sigma^{a}$ and by $\Phi^{*}$ the other electromagnetic potential. Then, instead of the above basis field, we may use the pseudo-orthogonal basis field, $\left\{\xi^{a}, \nabla^{a} \Psi, \nabla^{a} \Omega, \sigma^{a}\right\}$, where $\sigma^{a}$ denotes the unique null vector field for which $\sigma^{a} \nabla_{a} \Phi=-1$ and $\sigma^{a} \xi_{a}=\sigma^{a} \nabla_{a} \Phi^{*}=0$ hold throughout.

Consider, now, the last term of the right hand side of eq. (27). It can easily be checked

$$
\nabla^{a}\left[2 v^{-2} \xi_{(a} \Sigma_{b)}\right]=v^{-2}\left\{\xi_{a} \nabla^{a} \Sigma_{b}+\Sigma_{a} \nabla^{a} \xi_{b}+\xi_{b} \nabla^{a} \Sigma_{a}-2 v^{-1} \xi_{b} \Sigma_{a}\left(\nabla^{a} v\right)\right\}
$$

On the other hand, $\nabla^{a}\left[2 v^{-2} \xi_{(a} \Sigma_{b)}\right]$ can uniquely be decomposed, with regard to the basis field $\left\{\xi^{a}, \nabla^{a} \Psi, \nabla^{a} \Omega, \widehat{\Sigma}^{a}\right\}$ where $\widehat{\Sigma}^{a}$ denotes either $\sigma^{a}$ or $\Sigma^{a}$ according to that $\Sigma^{a}$ is null or not, as

$$
\nabla^{a}\left[2 v^{-2} \xi_{(a} \Sigma_{b)}\right]=\alpha \xi_{b}+\beta\left(\nabla_{b} \Psi\right)+\gamma\left(\nabla_{b} \Omega\right)+\delta \widehat{\Sigma}_{b}
$$

Then, with the aid of eq. (31), using the fact that $\xi^{a}$ is a Killing field, and the orthogonality properties of the vector system $\left\{\xi^{a}, \nabla^{a} \Psi, \nabla^{a} \Omega, \widehat{\Sigma}^{a}\right\}$, we find

$$
\begin{gathered}
\alpha=v^{-2}\left(\nabla^{a} \Sigma_{a}-v^{-1} \Sigma_{a} \nabla^{a} v\right), \\
\delta=0 .
\end{gathered}
$$

Moreover, a rather lengthy and tedious calculation also yields

$$
\begin{gathered}
\beta=-v^{-2} \omega_{a} \nabla^{a} \Psi \\
\gamma=v^{-2} \omega_{a} \nabla^{a} \Omega .
\end{gathered}
$$

Hence, as a direct consequence of eqs. (31) - (35), we obtain

$\nabla^{a}\left[2 v^{-2} \xi_{(a} \Sigma_{b)}\right]=v^{-2}\left[\left(\nabla^{a} \Sigma_{a}-v^{-1} \Sigma^{a} \nabla_{a} v\right) \xi_{b}+\left(\omega_{a} \nabla^{a} \Omega\right)\left(\nabla_{b} \Psi\right)+\left(\omega_{a} \nabla^{a} \Omega\right)\left(\nabla_{b} \Omega\right)\right]$ 
which implies along with eq. (27)

$$
\begin{aligned}
& \nabla^{a} T_{a b}=\frac{v^{-1}}{4 \pi}\{ {\left[\nabla^{a} \nabla_{a} \Psi-v^{-1}\left\{\left(\nabla^{a} \Psi\right)\left(\nabla_{a} v\right)+\omega_{a} \nabla^{a} \Omega\right\}\right]\left(\nabla_{b} \Psi\right) } \\
&\left.+\left[\nabla^{a} \nabla_{a} \Omega-v^{-1}\left\{\left(\nabla^{a} \Omega\right)\left(\nabla_{a} v\right)-\omega_{a} \nabla^{a} \Psi\right\}\right]\left(\nabla_{b} \Omega\right)+\nabla^{a}\left(v^{-1} \Sigma^{a}\right) \xi_{b}\right\} .
\end{aligned}
$$

Since the vectors $\xi^{a}, \nabla^{a} \Psi$ and $\nabla^{a} \Omega$ are linearly independent in the selected region $\nabla^{a} T_{a b}=0$ implies that eqs. (22) and (23) must be satisfied. This observation completes the proof of our proposal.

Finally, we would like to emphasize that whenever $\nabla^{a} \Psi$ and $\nabla^{a} \Omega$ are linearly independent the twice contracted Bianchi identity gives an additional restriction on the possible behaviour of electromagnetic fields. Clearly, the above argument also yields that the coefficient of $\xi_{b}$ in eq. (37) has to vanish identically, which means that the vector field, $J^{a}=v^{-1} \Sigma^{a}$, is divergence free. Note, that the presence of this conserved current is consistent with the general result, namely, if a spacetime admits a Killing vector field, $\xi^{a}$, then eq. (2) can be integrated to the conservation law

$$
\nabla^{a}\left(T_{a b} \xi^{b}\right)=0
$$

For the particular matter field considered in this paper the conserved current, $J^{a}$, can be shown to be proportional, for the case of a timelike Killing field, to the Poynting vector measured by observers following the Killing orbits. When, in turn, $\xi^{a}$ is, for example, an axial Killing field the current is proportional to one of the flows of the angular momentum of the electromagnetic field. In both cases the divergence free character of $J^{a}$ leads to the conclusion that there is no net flux of the relevant current through any closed surface.

\section{References}

[1] A. Trautman: Foundation and Current Problems of General Relativity, in Lectures on General Relativity, (Brandeis Summer Institute in Theoretical Physics, Prentice - Hall, Inc. Englewood Cliffs, New Yersey, 1965)

[2] R. M. Wald: General Relativity, (The University of Chicago Press, Chicago, 1984)

[3] B. K. Harrison: J. Math. Phys. 9, 1744 (1968)

[4] D. Kramer, H. Stefani, M. MacCallum and E. Herlt: Exact solutions of Einstein's equations (Cambridge; Cambridge University Press, 1980) 